\title{
Role of the gut microflora in metabolism of lipids and sterols
}

\author{
By H. Eyssen, The Rega Institute, 3000 Leuven, Belgium
}

The results of studies on gnotobiotic and conventional animals indicate that intestinal bacteria interfere with absorption of nutrients. The microflora could exert this undesirable activity by a direct action on the gut wall or the gut function, as well as by transformation of a variety of exogenous and endogenous substances into unabsorbable or potentially toxic compounds. This short review provides examples of the effect of intestinal bacteria on metabolism of lipids and sterols.

\section{Bile acids}

Bile acids are quantitatively the most important end-products of cholesterol metabolism. They arise from cholesterol by oxidation and removal of the terminal isopropyl fragment of the side chain, inversion of the $3 \beta$-hydroxyl group, $5 \beta$ saturation of the 5,6-double bond, and hydroxylation at either $\mathrm{C}_{7}, \mathrm{C}_{7}+\mathrm{C}_{12}$, or $\mathrm{C}_{6}+\mathrm{C}_{7}$ (Danielsson, 1963). After conjugation with taurine or glycine, these 'primary' bile acids are secreted in the bile.

Germ-free animals excrete only conjugated primary bile acids in bile and faeces (Gustafsson, Bergström, Lindstedt \& Norman, 1957; Kellogg \& Wostmann, I969). In conventional animals, however, the primary bile acids are subjected to deconjugation and dehydroxylation by microbial enzymes in the intestine, thus yielding the 'secondary' bile acids. The latter are at least partly reabsorbed and can again be transformed in the liver. The final result is a complex mixture of primary and secondary bile acids.

Many aerobic and anaerobic intestinal bacteria deconjugate bile acids (Midtvedt $\&$ Norman, 1967). In healthy subjects, deconjugation mainly takes place in the distal ileum, the caecum and the colon, not in the proximal small intestine. However, where there is overgrowth of the upper small intestine by a faecal microflora, such as in the stagnant loop syndrome, extensive deconjugation of bile acids in the jejunum could result in a deficiency of conjugated bile acids and in malabsorption of fats and other nutrients (Kim, Spritz, Blum, Terz \& Sherlock, r966; Tabaqchali \& Booth, 1966). Free bile acids, indeed, are less efficient in forming mixed micelles at the $\mathrm{pH}$ of the intestinal contents (Hofmann \& Borgström, 1962). Moreover, free bile acids could be absorbed from the upper small intestine by non-ionic diffusion (Dietschy, Salomon \& Siperstein, I966), whereas absorption of conjugated bile acids does not occur before the chyle reaches the lower segments of the ileum. 
In the lower intestinal tract, bile acids are oxidized or dehydroxylated. Microbial 7-dehydroxylation yields deoxycholic acid, lithocholic acid and numerous other metabolites (Drasar, Hill \& Shiner, i 966 ; Midtvedt \& Norman, 1967). In rats, deoxycholic acid is 7 -rehydroxylated by the liver during the enterohepatic circulation (Bergström, Rottenberg \& Sjövall, 1953). Conversely, the rabbit liver is unable to rehydroxylate deoxycholic acid at the $7 \alpha$-position (Ekdahl \& Sjövall, 1955). Therefore, after a number of enterohepatic cycles the bile acid pool of the rabbit is almost completely transformed into deoxycholic acid although this bile acid is not produced by the liver. In humans, $7 \alpha$-dehydroxylating bacteria do not become established in the gut before the age of $12-18$ months. This explains why deoxycholic acid usually is not present in bile of younger infants. Lithocholic acid is not found in germ-free animals, whereas faeces of most conventional mammalian species contain appreciable amounts of microbially formed lithocholic acid. The latter is a toxic compound and provokes cholangiofibrosis of the liver in chicks (Leveille, Sauberlich \& Hunt, I962) and in rabbits (Holsti, 1962). In rats given a protein-deficient diet, lithocholic acid induces ductular cell proliferation and formation of gall-stones (Palmer, I965).

Recently, it was shown that the gut microflora also can alter the bile acid spectrum of the bile without concomitant accumulation of microbially formed bile acid metabolites. For instance, $\beta$-muricholic acid accounts for $50-75 \%$ of the bile acids in bile of germ-free rats (Kellogg \& Wostmann, 1969) and mice (Eyssen, Parmentier, Mertens \& De Somer, 1973). Conversely, $\beta$-muricholic acid accounts for less than $25 \%$ of the bile acids in bile of conventional rats and mice in which cholic acid is the predominating bile acid. So far, this shift from $\beta$-muricholic to cholic acid in conventional rats and mice is unexplained. The possibility cannot be excluded that bacterial metabolites directly affect the bile-salt synthesizing capabilities of the hepatocytes. Transformation of $\beta$-muricholic acid into an unabsorbable derivative, or complete decomposition of the bile acid molecule, are alternative hypotheses.

Another interesting feature of host-microflora interaction is the observation that, in germ-free rats and mice, the composition of the bile acids depends upon the age and sex of the animals (Eyssen, Parmentier, Mertens et al. 1973 ). The ratio of $\beta$-muricholic acid to cholic acid is twice as high in male germ-free mice as in females. In both sexes, the relative importance of $\beta$-muricholic acid increases with age. This phenomenon seems to be masked by the activities of the microflora since it is much less pronounced in conventional animals.

Intestinal bacteria also influence the turnover and the faecal excretion of bile acids. Gustafsson et al. (1957) observed that conventional rats eliminate $50 \%$ of a dose of ${ }^{14} \mathrm{C}$-labelled cholic acid in $2 \mathrm{~d}$, compared to $\mathrm{II}^{\circ} 4 \mathrm{~d}$ in germ-free rats. Germfree chicks and rats eliminate 30 to $40 \%$ less bile acids through the faeces than their conventional counterparts (Eyssen, Van Messom \& Van den Bosch, 1969; Kellogg \& Wostmann, 1969). Moreover, recent investigations by Sacquet (1971) demonstrated that the small intestine of germ-free rats contains at least twice the amount of bile acids as the small intestine of conventional rats. This, of course, could have important implications for the absorption of neutral sterols and fatty acids. 
Neutral sterols

Conventional animals compensate for the increased loss of bile acids by a faster rate of oxidative catabolism of cholesterol into bile acids. After administration of $\left[26-{ }^{14} \mathrm{C}\right]$-cholesterol, conventional rats eliminate approximately $50 \%$ more $\left[{ }^{14} \mathrm{C}\right]$ carbon dioxide in the expired air than germ-free rats (Wostmann, Wiech \& Kung, 1966). It has also been observed that conventional chicks, mice and rats, excrete 5o$100 \%$ more cholesterol through the faeces (Evrard, Hoet, Eyssen, Charlier \& Sacquet, 1964; Eyssen I966; Kellogg \& Wostmann, 1969). Conflicting results have been reported on the concentration of cholesterol in serum and liver of germ-free and conventional animals. Most investigators found slightly raised cholesterol concentrations in serum of germ-free chicks and rats (Danielsson \& Gustafsson, r959; Wostmann \& Wiech, 196r; Coates, Harrison \& Moore, 1965). In general, however, the total blood-liver cholesterol pool does not seem to be significantly altered, presumably because this pool is under homoeostatic control.

When fed on a cholesterol diet, germ-free chicks, mice, and rats accumulate approximately twice as much cholesterol in the blood and the liver as conventional animals fed and housed under similar conditions (Eyssen, 1966). This finding, together with the observation that germ-free animals eliminate less cholesterol through their faeces, has been interpreted to mean that in conventional animals, intestinal micro-organisms interfere with the efficiency of absorption of cholesterol. Whether the less efficient cholesterol absorption in conventional animals is due to impairment of the absorptive capabilities of the intestinal mucosa, to the smaller bile acid pool in the small intestine, or to the accelerated rate of passage of food through the intestine, remains to be established.

The cholesterol molecule is characterized by a trans-junction of the $A / B$ rings and a 5,6-double bond in the steroid nucleus. Plant sterols such as $\beta$-sitosterol and campesterol share these characteristics with cholesterol from which they differ only in the side chain. Germ-free animals excrete only unmodified cholesterol and plant sterols (Evrard et al. 1964; Gustafsson, Gustafsson \& Sjövall, r966; Kellogg \& Wostmann, 1969). In conventional animals, however, the 5,6-double bond in the steroid nucleus of cholesterol can be $5 \beta$-H hydrogenated to yield less well absorbed coprostanol. Similarly, the plant sterols are converted into their respective $5 \beta-\mathrm{H}$ derivatives.

Recently, a micro-organism responsible for $5 \beta-\mathrm{H}$ reduction of 5,6 -unsaturated sterols in the rat has been isolated in pure culture (Eyssen, Parmentier, Compernolle, De Pauw \& Piessens-Denef, 1973). It is a strictly anaerobic bacterium tentatively classified into the genus Eubacterium. When associated with gnotobiotic rats, this Eubacterium converts more than $50 \%$ of cholesterol and plant sterols present in the caecum and the large intestine into coprostanol and $5 \beta$-H saturated plant sterols. This, however, does not result in a significant rise of the faecal neutral sterol output. In vitro, the steroid-reducing Eubacterium also converts 5,6-unsaturated steroid hormones of the androsten and pregnen series into the respective $5 \beta$-androstan and $5 \beta$-pregnan derivatives. Whether biohydrogenation of steroid hormones also occurs in vivo has not yet been established. 


\section{Fatty acids}

Studies on germ-free and conventional rats and chicks have shown that the intestinal microflora also interferes with absorption of fats (Hoet \& Eyssen, I964; Eyssen \& De Somer, I967). Lipolysis, however, does not seem to be significantly affected by the gut microflora (Hoet \& Eyssen, 1964; Tennant, Reina-Guerra, Harrold \& Goldman, r969).

The faecal fatty acid pattern appears to be a function of the microbial activities in the caecum and the large intestine. In germ-free rats fed on a maize-oil diet, the faecal $\mathrm{C}_{18}$ fatty acids are essentially unsaturated, mainly oleic and linoleic acid, and reflect the composition of the dietary maize oil (Hoet \& Eyssen, r964). Conversely, conventional rats excrete significantly less unsaturated $\mathrm{C}_{18}$ fatty acids and significantly more stearic acid. In addition, conventional rats excrete several typical 'bacterial' fatty acid derivatives, such as hydroxy fatty acids or cyclopropane fatty acids.

Micro-organisms producing hydroxystearic acid from oleic acid were isolated by Thomas (1972) and by Pearson (1973). However, although earlier investigations clearly demonstrated that certain rumen bacteria hydrogenate unsaturated $\mathrm{C}_{18}$ fatty acids, fatty acid-reducing micro-organisms from the caecum and the colon of other mammalian species have not been grown in vitro until very recently. In this laboratory we developed a culture medium in which biohydrogenation of $\mathrm{C}_{18}$ fatty acids could be carried out in vitro by mixed cultures of micro-organisms from rat caecal contents. These mixed cultures transformed added oleic and linoleic acid almost quantitatively into stearic acid in less than $72 \mathrm{~h}$. From the mixed culture we isolated a pure strain of a strictly anaerobic Gram-positive bacterium which, in vitro and in vivo, converts linoleic acid into an octadecenoic acid (Eyssen, De Pauw \& De Somer, 1973). This micro-organism, however, is unable to convert the octadecenoic acid into stearic acid. So far, all attempts to isolate a pure culture of the stearic acidproducing micro-organism have failed.

It seems noteworthy that the pathway followed by the octadecenoic acid-producing bacterium in reducing one of the double bonds of linoleic acid appears to be similar to that followed by Butyrivibrio fibrisolvens, a linoleic acid-reducing micro-organism isolated from the rumen of sheep (Kepler, Hirons, McNeill \& Tove, I966). Both micro-organisms first convert linoleic acid into a linoleic acid isomer, presumably a conjugated isomer. Under appropriate conditions, for example an oxidationreduction potential below $-225 \mathrm{mV}$, one of the double bonds of the isomer is reduced to yield an octadecenoic acid (Kepler et al. I966; Eyssen, De Pauw et al. I973). This indicates that biohydrogenation of unsaturated $C_{18}$ fatty acids in the rumen of sheep and in the caecum of the rat follow similar pathways and, perhaps, are carried out by closely related bacterial species.

\section{Summary}

The results of comparative studies on gnotobiotic and conventional animals show that the gut microflora interferes with the metabolism of lipids and sterols. Intestinal bacteria transform the primary bile acids into a variety of secondary bile acids, reduce the bile acid pool in the intestine, promote the faecal excretion and accelerate the 
turnover of bile acids. This results in a faster rate of catabolism of cholesterol to bile acids. The intestinal microflora promotes the excretion of cholesterol through the faeces and converts 5,6 -unsaturated steroids into the $5 \beta-\mathrm{H}$ saturated derivatives. The gut microflora also interferes with the absorption of fats, produces bacterial fatty acids and converts linoleic and oleic acid into stearic acid by biohydrogenation of the double bonds in these $\mathrm{C}_{18}$ fatty acids.

\section{REFERENCES}

Bergström, S., Rottenberg, M. \& Sjövall, J. (1953). Hoppe-Seyler's Z. physiol. Chem. 295, 278.

Coates, M. E., Harrison, G. F. \& Moore, J. H. (1965). Ernährungsforschung 10, 251.

Danielsson, H. (1963). Adv. Lipid Res. I, 335.

Danielsson, H. \& Gustafsson, B. (1959). Archs Biochem. Biophys. 83, 482.

Dietschy, J. M., Salomon, H. S. \& Siperstein, M. D. (1966). F. clin. Invest. 45, 832.

Drasar, B. S., Hill, M. J. \& Shiner, M. (Ig66). Lancet i, 1237.

Ekdahl, P. H. \& Sjövall, J. (1955). Acta physiol. scand. 34, I.

Evrard, E., Hoet, P. P., Eyssen, H., Charlier, H. \& Sacquet, E. (I964). Br. F. exp. Path. 45, 409.

Eyssen, H. (1966). In Proc. Symposium on Gnotobiology, Int. Congr. Microbiol., Ix, Moscow, 1966, p. 329.

Eyssen, H., De Pauw, G. \& De Somer, P. (1973). In Germ-Free Research : Biological Effect of Gnotobiotic Environments p.277 [J. B. Heneghan, editor]. New York: Acadmic Press.

Eyssen, H. \& De Somer, P. (I 967). Poult. Sci. 46, 323.

Eyssen, H., Parmentier, G., Compernolle, F., De Pauw, G. \& Piessens-Denef, M. (1973). Eur. J. Biochem. 36, 411 .

Eyssen, H., Parmentier, G., Mertens, J. \& De Somer, P. (1973). In Germ-free Research : Biological Effect of Gnotobiotic Environments p.271 [J. B. Heneghan, editor]. New York: Academic Press.

Eyssen, H., Van Messom, G. \& Van den Bosch, J. ( I 969). In Germ-free Biology p.97 [E. A. Mirand and N. Back, editors]. New York: Plenum Press.

Gustafsson, B. E., Bergström, S., Lindstedt, S. \& Norman, A. (r957). Proc. Soc. exp. Biol. Med. 94, 467.

Gustafsson, B. E., Gustafsson, J. A. \& Sjövall, J. (1966). Acta chem. scand. 20, 1827.

Hoet, P. P. \& Eyssen, H. (I964). Gut 5, 309.

Hofmann, A. F. \& Borgström, B. (I962). Fedn Proc. Fedn Am. Socs. exp. Biol. 21, 43.

Holsti, P. (1962). Acta path. microb. scand. 54, 479.

Kellogg, T, F, \& Wostmann, B. S. (1969). F. Lipid Res. Io, 495.

Kepler, C. R., Hirons, K. P., McNeill, J. J. \& Tove, S. B. (1966). 7. biol. Chem. 24I, 1350.

Kim, Y. S., Spritz, N., Blum, M., Terz, J. \& Sherlock, P. (1966). F. clin. Invest. 45, 956.

Leveille, G. A., Sauberlich, H. E. \& Hunt, R. D. (r962). Poult. Sci. 41, r991.

Midtvedt, T. \& Norman, A. (I 967). Acta path. microbiol. scand. 71, 629.

Palmer, R. H. (1965). Science, N.Y. 148, 1339.

Pearson, J. R. (1973). Proc. Nutr. Soc. 32, 8A.

Sacquet, E. (197I). Flore microbienne du tractus digestif et nutrition. Thèse doctorat ès Sciences, University of Paris.

Tabaqchali, S. \& Booth, C. C. (1966). Lancet ii, 12.

Tennant, B., Reina-Guerra, M., Harrold, D. \& Goldman, M. (1969). F. Nutr. 97, 65.

Thomas, P. J. (1972). Gastroenterology 62, 430.

Wostmann, B. S. \& Wiech, N. L. (r96r). Am. F. Physial. 20r, г027.

Wostmann, B. S., Wiech, N. L. \& Kung, E. (1966). F. Lipid Res. 7, 77. 